\title{
Metrology of a mirror at the Advanced Photon Source: comparison between optical and $\mathrm{x}$-ray measurements
}

\author{
Lahsen Assoufid, Jonathan Lang, Jin Wang, and George Srajer \\ Advanced Photon Source \\ Argonne National Laboratory \\ 9700 South Cass Avenue, Argonne, Illinois 60439, USA \\ ABSTRACT
}

\begin{abstract}
This paper describes metrology of a vertically focusing mirror on the bending magnet beamline in sector-1 of the Advanced Photon Source, Argonne National Laboratory. The mirror was evaluated using measurements from both an optical long trace profiler and x-rays. Slope error profiles obtained with the two methods were compared and were found to be in a good agreement. Further comparisons were made between $\mathrm{x}$-ray measurements and results from the SHADOW ray-tracing code.
\end{abstract}

Keywords: long trace profiler, metrology, x-ray mirror, synchrotron radiation

\section{INTRODUCTION}

The performance of grazing-incidence mirrors used on beamlines at third-generation synchrotron radiation sources depends considerably on the magnitude of large scale irregularities (figure error) as well as on the smoothness (microroughness or finish) of their reflecting surfaces. Typically, surfaces slope errors and roughnesses better than $3 \mu \mathrm{rad}$ and $3 \AA \mathrm{rms}$, respectively, are required in order to preserve the brilliance of these sources. Considering the high cost of mirrors and their long delivery time, it is important to evaluate their optical quality upon the delivery from the manufacturer as part of the acceptance criteria. At the Advanced Photon Source (APS), this is normally done using optical interferometers available at the metrology laboratory. Furthermore, state-of-the-art optical metrology instruments have adequate sensitivity and cover a wide range of spatial frequencies to provide the user with useful information in order to predict the performance of optics. However, the ultimate tests are those performed at, or near, wavelengths at which the optic is intended to operate.

The submitted manuscript has been created by the University of Chicago as Operator of Argonne National Laboratory ("Argonne") under Contract No. W-31-109-ENG-38 with the U.S. Department of Energy. The U.S. Government retains for itself, and others actIng on its behalf, a paid-up, nonexclusive, irrevocable wortdwide license in said article

to reproduce, prepare derivative works, distribute copies to the public, and perform publicty and display publicly, by or on behalf of the Government. 


\section{DISCLAIMER}

This report was prepared as an account of work sponsored by an agency of the United States Government. Neither the United States Government nor any agency thereof, nor any of their employees, make any warranty, express or implied, or assumes any legal liability or responsibility for the accuracy, completeness, or usefulness of any information, apparatus, product, or process disclosed, or represents that its use would not infringe privately owned rights. Reference herein to any specific commercial product, process, or service by trade name, trademark, manufacturer, or otherwise does not necessarily constitute or imply its endorsement, recommendation, or favoring by the United States Government or any agency thereof. The views and opinions of authors expressed herein do not necessarily state or reflect those of the United States Government or any agency thereof. 


\section{DISCLAIMER}

Portions of this document may be illegible in electronic image products. Images are produced from the best available original document. 
This paper describes a metrological investigation of a particular mirror used to vertically focus the $\mathrm{x}$-ray beam on the bending magnet (BM) beamline in sector-1 of the APS. Comparison was made between slope error profiles obtained with the long trace profiler and $\mathrm{x}$-rays tests conducted during the commissioning of this beamline. The mirror, which is a permanent component of the beamline, is made of a long flat Zerodur substrate that is $1020 \mathrm{~mm}$ long, $65 \mathrm{~mm}$ wide, and $60 \mathrm{~mm}$ high, and is bendable with a radius from infinity (flat shape) to about $3 \mathrm{~km}$ (a cylinder). The reflecting surface is coated with a $750-\AA-$ thick layer of palladium (with a $\sim 50 \AA$ chromium buffer layer), and was specified to have a $3 \AA$ rms roughness and $\leq 5 \mu \mathrm{rad}$ rms slope error. The beam image from the mirror recorded on a CDD camera (with the mirror fully illuminated) was found to exhibit a series of parallel horizontal stripes. This paper summarizes both $\mathrm{x}$-ray and optical studies of the mirror to determine the source of the stripes.

\section{OPTICAL MEASUREMENTS OF SURFACE PROFILE}

The optical measurements were carried out using the long trace profiler (LTP) at the metrology laboratory of the APS. This laboratory provides characterization of optical components primarily on beamlines at the APS. (A detailed description of the this laboratory and its instrumentation can be found in reference 1.)

\subsection{Description of Long Trace Profiler}

The LTP is a non-contact optical profiling instrument used for measurement of surface slope and figure errors on large grazing incidence flat or aspheric mirrors such as those used at synchrotron radiation sources. ${ }^{2}$ The LTP at the APS was designed to handle mirrors up to two meters in length. It can characterize surface height profiles over spatial periods ranging from $2 \mathrm{~m}$ (the maximum scan length) to 2 $\mathrm{mm}$ (the Nyquist period for 1-mm sampling). It complements the other instruments available at the APS metrology laboratory. Modification to the original LTP design has considerably improved the accuracy and repeatability of the instrument. ${ }^{3}$ A standard deviation of $0.3 \mu \mathrm{rad}$ with a corresponding error bar height of $4.6 \mathrm{~nm}$ was obtained from an average of 10 profiles, measured of a $500 \mathrm{~mm}$ long mirror with a $5 \mathrm{~km}$ radius of curvature. ${ }^{3}$

\subsection{Slope Error Measurement with the LTP}

The mirror measurements consisted of a series of 10 slope error scans taken along the mirror centerline. Each scan was $990 \mathrm{~mm}$ in length containing 545 points at a 2-mm sampling rate. The mirror was scanned unmounted (i.e., without the bending mechanism). It was put face up and was simply supported at the bottom face near the ends. Therefore, the raw surface profile contains deflection due to gravity. The residual profile was obtained by subtracting the tilt and the best fit cylinder from the raw profile. The resulting slope error profile (see next section) led to a $4.9 \mu \mathrm{rad}$ rms slope error and $7 \mathrm{~km}$ radius. 


\section{X-RAY SLOPE ERROR PROFILE MEASUREMENTS}

As mentioned earlier, the $\mathrm{x}$-ray measurements were performed during the commissioning of the BM beamline. The mirror was attached to its bending mechanism and was mounted to deflect the beam downward. The bender mechanism is a four-point scheme and is actuated pneumatically. During the tests, various aspects of the mirror were investigated including its reflectivity, focusing properties, and surface profile. This paper focuses on the profile and topography of the reflecting surface.

\subsection{Experimental Set-up and Measurement Method}

The $\mathrm{x}$-ray experimental setup is shown in Figure 1. It consists of radiation from a bending magnet source, a system of vertical and horizontal slits, a collimating mirror, a double-crystal monochromator, a set of narrow vertical slits (beam defining slits), the test mirror, and finally a CCD camera for recording the intensity profile of the reflected $x$-ray pencil beam.

The source size (FWHM) is $0.3 \mathrm{~mm} \times 0.3 \mathrm{~mm}$. The maximum accepted horizontal and vertical angular openings are limited to 3.7 and $0.17 \mathrm{mrad}$, respectively. The first set of slits limits the beam size to $3 \mathrm{~mm}$ horizontally and $2 \mathrm{~mm}$ vertically. The collimating mirror has three functions: it vertically collimates the beam, acts as a low-pass power filter for the white radiation from the BM source, and acts as a higher harmonic rejecter. The reflected beam is monochromatized to $10 \mathrm{keV}$ (1.239 $\AA$ wavelength) by the double-crystal silicon (Si) (111) monochromator. The narrow vertical slits located $37.8 \mathrm{~m}$ downstream from the monochromator limit the beam height to $200 \mu \mathrm{m}$ at the CCD camera position. Because of the use of a monochromatic beam, the size of these vertical slits was chosen to be much larger than the transverse coherence length of the bending magnet source at $1.239 \AA$ wavelength, so that the measurements are based on pure ray tracing.

The transverse coherence length can be expressed as $l_{t}=\lambda D / 2 \sigma$, where $\sigma$ is the source size, $\lambda$ is the radiation wave length, and $D$ the distance from source to slits. With the current setup, the effective value of $D$ through the collimating mirror is $25.5 \mathrm{~m}$, the transverse coherence length is about $53 \mu \mathrm{m}$.

The mirror being tested is located $45.5 \mathrm{~m}$ from the source and was set to deflect the $\mathrm{x}$-ray beam downward at a $5.6 \mathrm{mrad}$ angle, which gives a beam footprint of about $35 \mathrm{~mm}$ on the surface of the mirror. The detector is located about $10 \mathrm{~m}$ from the test mirror and consists of a home-made CCD camera with an area of $1 \mathrm{~cm} \times 1 \mathrm{~cm}$ (corresponding to $512 \times 512$ pixels, and $19 \mu \mathrm{m} \times 19 \mu \mathrm{m}$ pixel size.) 
The measurement method consisted of scanning the mirror surface with a fine, well-collimated, monochromatic pencil beam at grazing-incidence angle. The specularly reflected beam hits the CCD camera placed at some distance from the test mirror. A scintillating material is used to convert the $\mathrm{x}$-rays to visible photons. The mirror is translated vertically, and the intensity and position of each spot is recorded vs. the mirror position. The relative location of the centroid of each recorded intensity profile gives a measure of the local slope error and is displayed vs. position on the mirror surface. The resulting profile (corrected for the mirror translation and tilt) gives the slope deviation profile of the mirror surface.

\subsection{Measurements and Results}

The measurements were carried out with the unfocused mirror (i.e., no bending moment was applied to the mirror). The mirror surface was scanned across the incident beam by vertically translating the mirror table. In this fashion, the beam, which was incident on the mirror, hits the same spot on the optics that preceded it, thus keeping the same beam quality during the entire scanning operation. The table is actuated by three precision translation stages with a minimum vertical step size of $0.635 \mu \mathrm{m}$. The accuracy of the translation stage was measured and was found to be in the order of $25 \mu \mathrm{m}$ over $300 \mathrm{~mm}$ travel, i.e., about $0.0083 \%$. The total travel required to scan the mirror length is about $5.2 \mathrm{~mm}$. This translates into a maximum slope error of $+/-0.43 \mu \mathrm{rad}$ over the $1.020 \mathrm{~m}$ mirror length. This error is comparable to the LTP repeatability.

Figure 2 compares the measured $x$-ray slope error profile with the LTP measurements. The table below compares statistical data. The trace length for the $\mathrm{x}$-ray profile contains 292 points with a $4.71-\mathrm{mm}$ increment along the mirror length, while that of the LTP has 545 points with a 2-mm sampling rate. Both results are for profiles corrected for tilt as well as curvature due to the effect of gravity.

\begin{tabular}{|l|l|l|}
\hline Measurement & $\begin{array}{l}\text { Root-mean square } \\
(\mu \mathrm{rad})\end{array}$ & Peak-to-valley $(\mu \mathrm{rad})$ \\
\hline LTP & 4.9 & 38.5 \\
\hline x-rays & 6.3 & 46.6 \\
\hline
\end{tabular}

\subsection{Discussion of Results}

As one can see from Figure 2, the two residual slope error profiles correlated identical features, and the statistical values agree within $17-22 \%$. The bumps at the edges are due to the manufacturer's grinding and polishing techniques. 
The close similarity between the two profiles also shows that the error introduced by the mirror vertical translation stage of the mirror was not significant enough to be detected. The slight relative tilt in the two profiles (particularly within the central portion) is believed to be due to the way the mirror was supported during the measurements and the way the data were processed. During the LTP measurements, the mirror was supported at the bottom face (mirror facing upward) near the ends, while during the $x$-ray measurements, the mirror was mounted to the bender and the exact shape of the mirror at unfocused (nominally flat) position is unknown.

Usually, mirror-bender assemblies are calibrated using the long trace profiler before installation on a beamline. During LTP measurements, the radius of curvature is recorded vs. the bender encoder reading, in order to generate a calibration curve. Here, because the bender support structure of the mirror did not allow for LTP characterization, optical calibration of the bender was not done. Therefore, the mirror surface profile when the mirror surface in nominally flat is unknown. However, the measured $x$-ray profile indicates that the mirror is slightly constrained by the bending mechanism (presence of second-order term in the measured profile). The constraint could explain the difference at the right end of the two profiles (Figure 2a) where the supporting points are located.

Figure $2 \mathrm{~b}$ shows a better agreement between the two profiles when only the $820-\mathrm{mm}$ portion of the mirror is considered, and the residual curvature is removed from that portion of the LTP data.

The difference in the finer details stems from the fact that the $\mathrm{x}$-ray profile was obtained with a much lower resolution than was the LTP measurement. The $\mathrm{x}$-ray beam footprint on the mirror surface was about $35 \mathrm{~mm}$. Therefore, the local slope error measurement had an effective resolution of about $35 \mathrm{~mm}$. However, to obtain a well-defined slope error profile, data were taken with a 4.71-mm increment, which obviously led to a large overlap between two consecutively measured areas. Although the resolution was much larger than the step size, we can see finer features on a length scale much smaller that the resolution value at each step. We should emphasize the purpose of this experiment was merely a qualitative comparison between LTP measurements and mirror performance in the actual operating environment. A more accurate study can be done by performing high-resolution measurements using a pink or white beam (a broader bandwidth) rather than monochromatic radiation, which will allow one to use a much narrower pencil beam. Such technique has been implemented at the ESRF ${ }^{4,5}$ Slope error measurements were obtained with 5-mm lateral resolution, and precision and accuracy better than $25 \mathrm{nrad}(\mathrm{rms})$ and $50 \mathrm{nrad}$, respectively. ${ }^{5}$ 


\section{MIRROR SURFACE TOPOGRAPHY}

The surface topography of the mirror was also recorded using a CCD camera placed at $10 \mathrm{~m}$. In this case, the mirror was set at $2.8 \mathrm{mrad}$ grazing-incidence angle on the beamline, and the surface topography was measured fully illuminated with the incident monochromatic beam.

The images were found to exhibit a series of a parallel stripes along with some randomly oriented intense lines. The randomly oriented intense lines are due to defects on the CCD camera scintillator, but the series of parallel stripes were found to arise almost entirely from the surface structure of the vertically focusing mirror (small contributions were expected from the collimating mirror and the double-crystal monochromator.)

To make sure the observed stripes were not a contribution from other optical elements, intensity profiles were recorded at various wavelengths and mirror bending radii. Figure 3 shows the recorded image at $13.5 \mathrm{keV}$. The stripes were consistently found in all of the measurements. The spacing between the stripes remains constant as the radiation wavelength is changed and shrunk or expanded correspondingly as the mirror radius was varied.

To further verify that these stripes arose from the figure errors of the mirror, a simulation of the response of the mirror surface under an incident $\mathrm{x}$-ray beam was performed using the ray-tracing code SHADOW. ${ }^{6}$ To do so, we used the measured LTP profile (shown in Figure 2) to generate a 3-D surface profile of the mirror, then the ray tracing was conducted using a uniform rectangular source that has a total of 5000 rays. The LTP slope error profile was first converted into the corresponding height profile, then the SHADOW-usable surface file was generated using the SHADOW "PRESURFACE" routine. Because of the limited array dimensions available, only the central portion of the mirror was modeled.

The generated image of the mirror surface is displayed in Figure 4. It exhibits stripe structure similar to that observed with $\mathrm{x}$-rays. This shows that the observed stripes are indeed of geometrical origin, and that even optical surfaces with slope errors as small as $5 \mu \mathrm{rad}$ rms can affect the spatial profile of the $x-$ ray beam.

\section{CONCLUSIONS}

This study shows that measurements performed with the APS LTP compare well with $x$-ray profilometry. The LTP is therefore, a valuable tool for characterizing and partially predicting the 
performance of synchrotron radiation mirrors. The topography and the SHADOW simulation showed that even optical surfaces with slope errors as small as $5 \mu \mathrm{rad}$ rms can affect the spatial profile of the $\mathrm{x}$-ray beam. This also asserts that specifying a mirror with a single statistical (rms) value is not sufficient, and, depending on the application, other criteria such as a the power spectral density function should also be considered.

\section{ACKNOWLEDGEMENTS}

One of the authors (L. Assoufid) would like to thank Drs. Albert Macrander, Dennis Mills and Jean Susini (ESRF, Grenoble, France) for useful discussions. This work is supported by the U. S. Department of Energy, BES-Materials Sciences, under contract No. W-31-109-ENG-38.

\section{REFERENCES}

1. C. Bresloff and D. Mills, “The Advanced Photon Source Metrology Laboratory," Rev. Sci. Instrum. 67 (9), (1996) CD ROM

2. P. Z. Takacs, S.-N. Qian, and J. Colbert, "Design of a long-trace profiler," In Metrology-Figure and Finish, Bruce Truax, ed., Proc. SPIE vol. 749, 59-64 (1987)

3. P.Takacs, Eugene L. Church, Cynthia Bresloff, Lahsen Assoufid, "Long Trace Profiler Measurement Repeatability Improvements," submitted to Appl. Optics

4. Ph. Gondoin, A. Freund, S. Gougeon, D. de Chambure, K. van Katwijk, D. Labergerie, U. Lienert, N. Schulz, Proc. SPIE vol. 2515, 70-83 (1987)

5. Olivier Hignette, Andreas Freund and Elia Chinchio, "incoherent x-ray mirror surface metrology," Proc. SPIE, vol. 3152, 188-199 (1997)

6. F. B. Lai and F. Cerrina, Nucl. Instrument and Meth. A246, 337 (1986) 


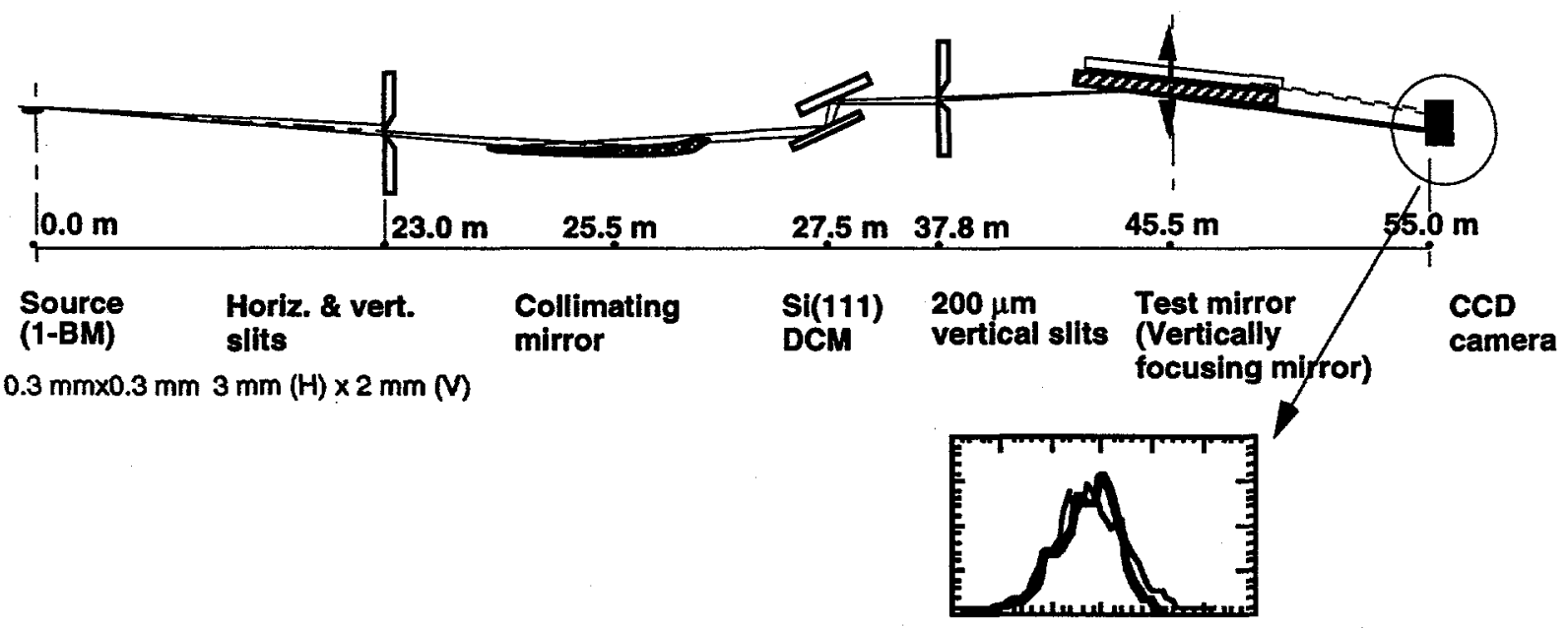

Figure 1: The experimental setup for measuring the $x$-ray slope error profile of a vertically focusing mirror on the bending magnet beamline in sector-1 at the APS. 
a)

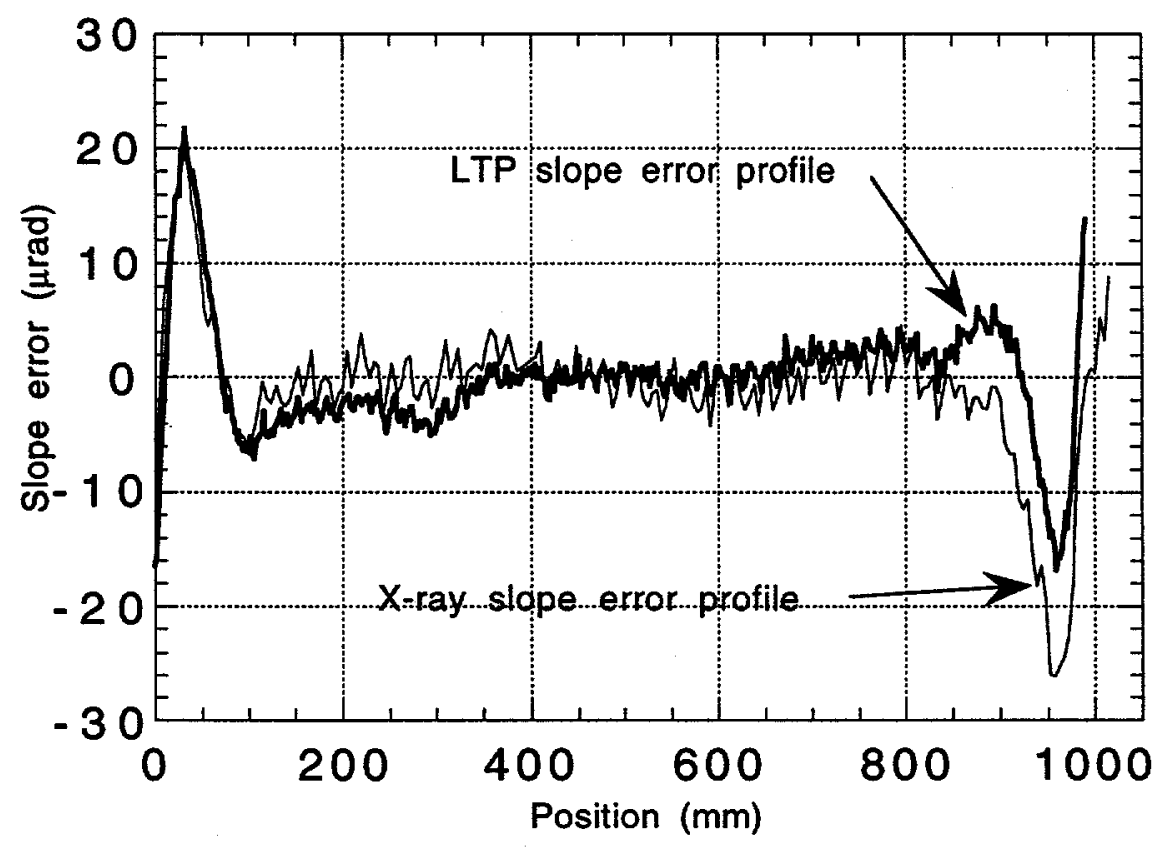


b)

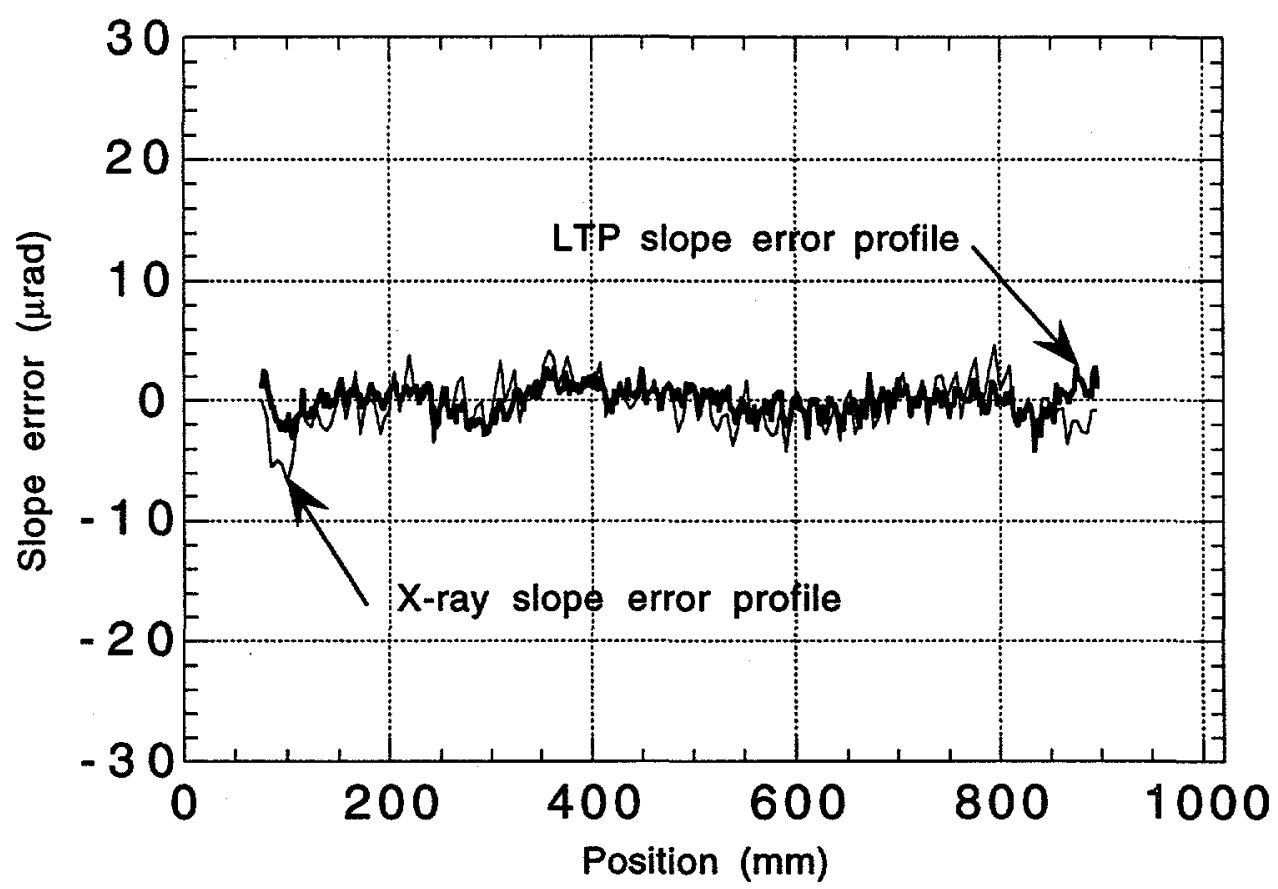

Figure 2: Surface slope error profile of the vertically focusing mirror on the bending magnet (BM) beamline in sector-1 of the APS: comparison between the LTP profile and the synchrotron radiation x-ray measurements over a) the entire mirror length, b) the central $820 \mathrm{~mm}$ trace length. The best fit radius was subtracted from the LTP profile within this portion of the mirror. The two profiles show almost identical features. 


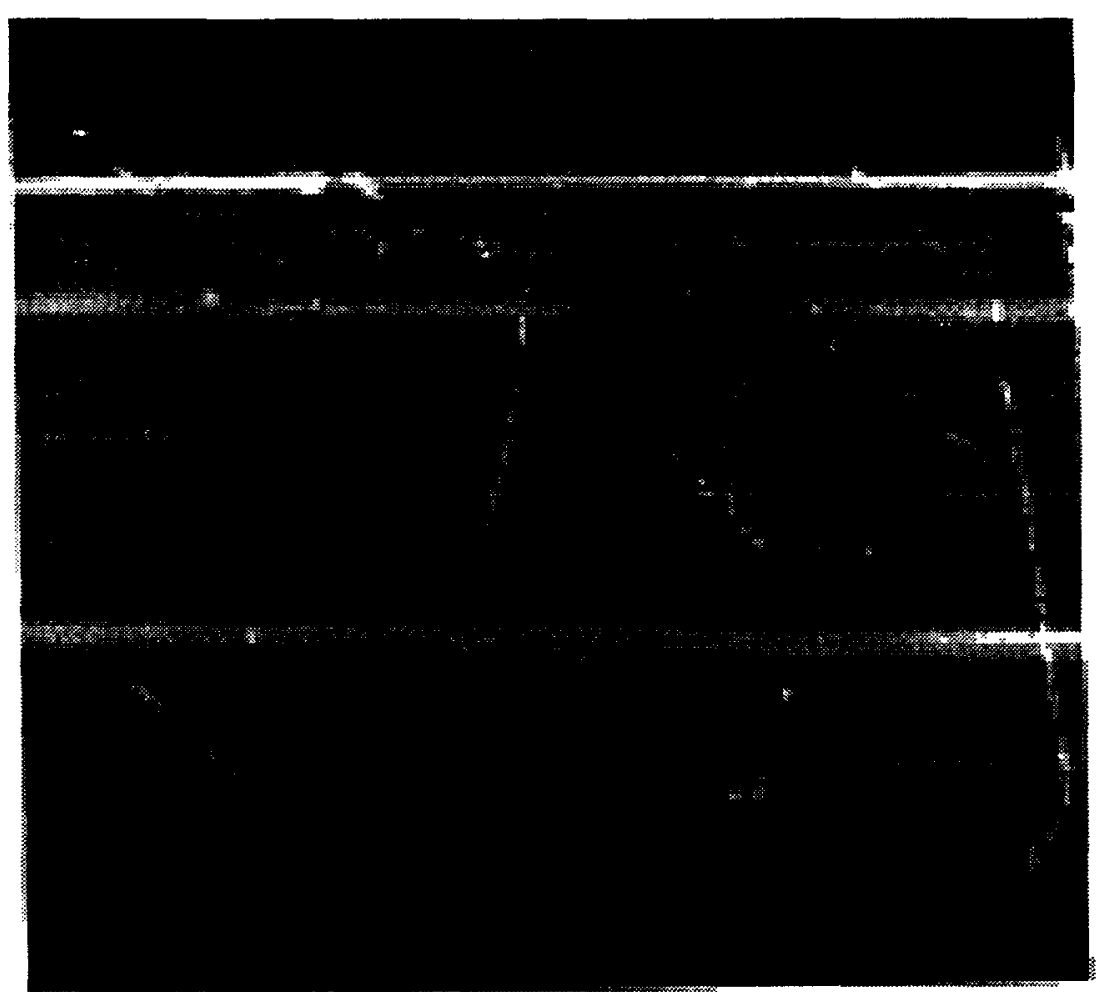

Figure 3: Topograph of the mirror obtained with BM radiation. The mirror was set flat at a $2.8 \mathrm{mrad}$ angle, and its surface was fully illuminated with the incident monochromatic x-ray beam. The x-ray image was collected using a CCD camera. The randomly oriented intense lines are due to defects in the CCD camera scintillating crystal, but the series of parallel stripes arise from the mirror surface figure errors. Small contributions to this structure were expected from the collimating mirror and the doublecrystal monochromator. 


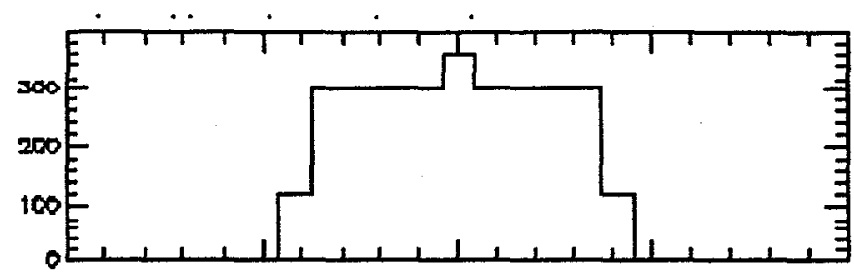

Prepared:

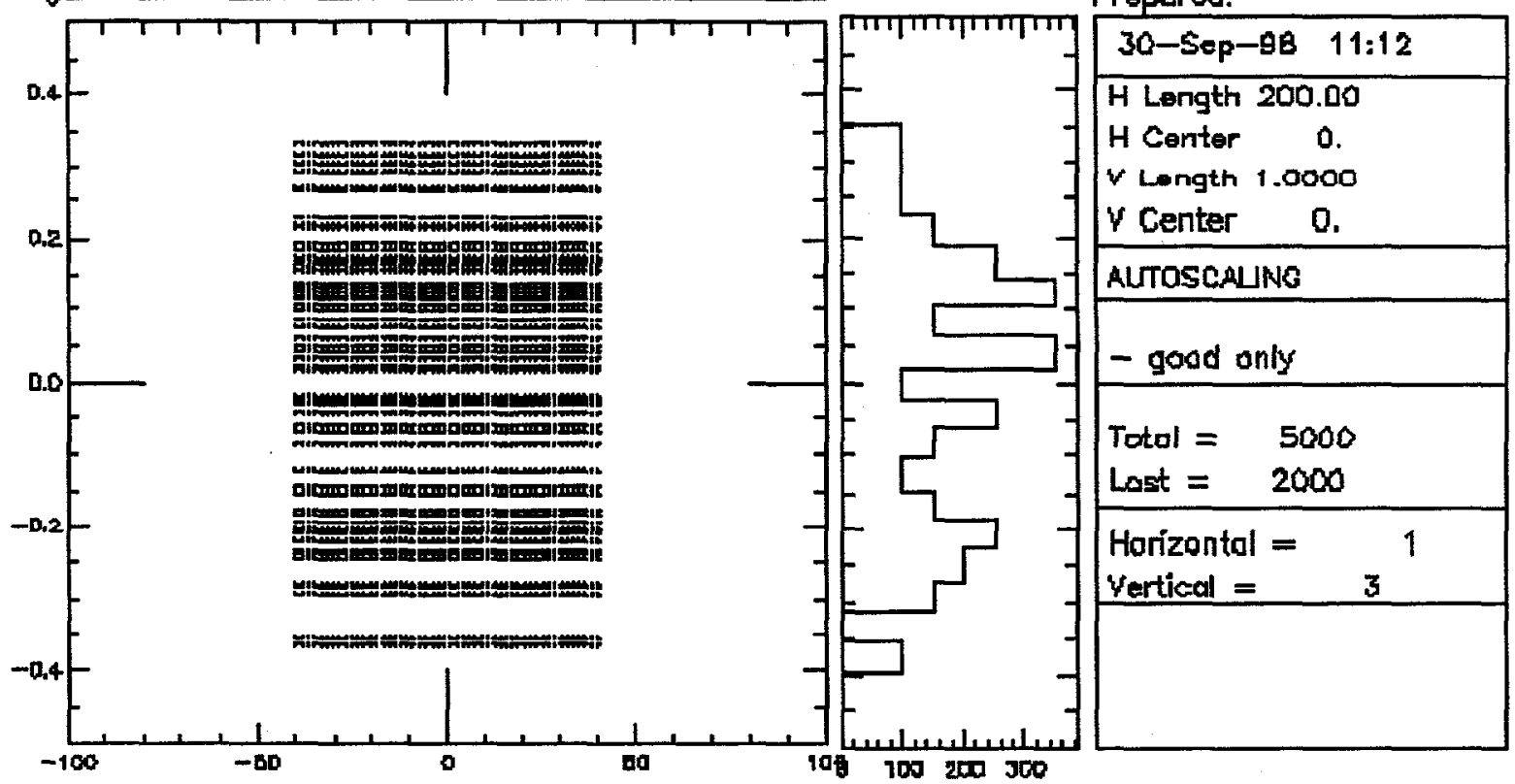

Figure 4: Simulated image using the LTP height profile as input using the SHADOW ray-tracing code. A rectangular uniform source was used. The simulated image shows a parallel stripe structure similar to that observed in the $\mathrm{x}$-ray topography measurements. 